\title{
DNA Melting in Aggregates: Impeded or Facilitated?
}

\author{
A. G. Cherstvy*,†, and A. A. Kornyshev \\ Institut für Festkörperforschung, Theorie-II, Forschungszentrum Jülich, 52425 Jülich, Germany, and \\ Department of Chemistry, Faculty of Physical Sciences, Imperial College London, \\ SW7 2AZ London, United Kingdom
}

Received: March 4, 2005; In Final Form: May 4, 2005

\begin{abstract}
How does DNA melt in columnar aggregate relative to its melting in diluted solution? Is the melting temperature increased or decreased with the aggregate density? Have DNA-DNA interactions, predominantly of electrostatic nature, an effect on the character of the melting transition? In attempt to answer these questions, we have incorporated the theory of electrostatic interactions between DNA duplexes into the simplest model of DNA melting. The analysis shows that the effect of aggregate density is very different for aggregates built of homologous (or identical) DNA fragments relative to the case of DNA with random base pair sequences. The putative attraction between homologous DNA helices hampers their melting and increases the melting temperature and can even dramatically change the character of the transition. In the aggregate of nonhomologous DNAs, the pattern of electrostatic interactions is more complicated, and their effect could be opposite; in some cases we may even expect electrostatically induced melting. These findings define new directions for melting experiments in dense DNA assemblies.
\end{abstract}

\section{Introduction}

At ambient conditions, DNA adopts the double-helical B form. ${ }^{1}$ With an increase of temperature, it starts to melt, as hydrogen bonds between base pairs (bp's) begin to rupture. There are several ways to detect this; ${ }^{2}$ some of them use the more pronounced adsorption of 260-nm UV light by bp's on unzipped DNA single strands. ${ }^{3,4}$ Major changes in DNA structure occur close to the melting temperature, $T_{\mathrm{m} 0}$, which typically lies between 40 and $100{ }^{\circ} \mathrm{C}$, depending in particular on the bp content and salt conditions. The process of melting is not abrupt but is reminiscent of a glass transition; $T_{\mathrm{m} 0}$ is defined as the temperature at which half of all bp's are melted. A partially melted long DNA is a disordered sequence of alternating melted and intact/helical domains.

Usually, DNA melting is studied in dilute solutions, where various factors affect DNA stability. The melting temperature shifts downward with the decrease in salt concentration, $n_{0},{ }^{5}$ or the decrease in DNA GC content. The melting can be facilitated by DNA stretching, ${ }^{6}$ twisting, ${ }^{7}$ of unzipping ${ }^{8}$ and by the presence of divalent transition metal cations in solution. ${ }^{9-11}$

Several theories have been developed for the description of melting or the helix - coil transitions of biopolymers, ${ }^{12,13}$ DNA in particular. ${ }^{14-16}$ The impact of many biologically relevant factors has been elucidated, including the effect of electrostatic repulsion between the strands of the same DNA. ${ }^{13,17}$ Below, we discuss the effect of interactions between DNAs on DNA melting in assemblies. To our knowledge, this question has not been theoretically studied before in detail, although a phenomenological account for intermolecular coupling has been pro-

* Author to whom correspondence should be addressed. E-mail: cherstvy@ mpipks-dresden.mpg.de.

$\dagger$ Forschungszentrum Jülich.

$\div$ Imperial College London.

$\S$ Present address: Max-Planck-Institut für Physik komplexer Systeme, Nöthnitzer Stra $\beta$ e 38, 01187 Dresden, Germany. posed. ${ }^{18}$ This paper presents a preliminary, simplified, and in parts speculative analysis intended to provoke new experiments.

\section{Electrostatic Zipper}

The B-DNA duplex is a highly charged molecule; its $10 \mathrm{bp}$ per helical turn deliver 20 charges on the phosphate groups. A large portion of these charges is compensated for by adsorbed/ condensed counterions. (Many of them prefer to reside in the grooves.) This basically determines the helical motif of the distribution of negative and positive charges along DNA. Together with the Debye screening in the electrolyte, these motifs cause nearly exponential decay of DNA-DNA electrostatic interactions with interaxial separation $R$ between the duplexes, in accordance with experimental observations. ${ }^{19,20}$

In ref 21 , the interaction energy between two parallel DNA duplexes has been calculated as an exact solution of the linear Poisson-Boltzmann equation. The helicity of the distribution of DNA phosphates and of adsorbed cations was considered explicitly. This theory has rationalized a number of phenomena related to DNA-DNA interaction. ${ }^{22-24}$ It predicts, in particular, that a zipperlike attraction between juxtaposed DNAs should take place, caused by an attraction of the phosphates of strands of one DNA with cations adsorbed in the grooves of another DNA. ${ }^{25}$ Binding of cations in the DNA major groove enhances this attraction. ${ }^{25}$

In the first variant of this theory, DNAs were considered as ideal double helices. However, DNA is not a perfect "spiral staircase". The twist angles between particular bp's vary about the mean value of $\sim 34^{\circ}$ by, on average, $\pm 4-6^{\circ}$. (The actual deviation depends on the bp sequence.) These twist-angle distortions have been taken into account in the extension of the theory. ${ }^{26}$ They have no effect on the interaction of two identical DNAs, because the patterns of their twist-angle "distortions" are the same. However, if the opposing DNA "texts" are different, as in the case of nonhomologous sequences, then the effect of helix nonideality becomes dramatic for sufficiently long 
sequences. ${ }^{26}$ Indeed, if the helices were torsionally rigid, then this helical nonideality would have accumulated as a random walk, inevitably disrupting the zipperlike strand-to-groove alignment over the helical coherence length $\lambda_{\mathrm{c}}=h /\left\langle\Delta \Omega^{2}\right\rangle$. For helical rise $h \approx 3.4 \AA$ and typical mean deviation from the average twist angle $\sqrt{\left\langle\Delta \Omega^{2}\right\rangle} \approx 0.07-0.1 \mathrm{rad},{ }^{27-29} \lambda_{\mathrm{c}} \approx 300-$ $700 \AA$ A. This disruption effect, nonlinearly depending on the DNA sequence length, would impede or disable the electrostatic zipper. $^{26}$

However, only short fragments of DNA could be considered torsionally rigid. For this reason, DNA helical nonideality and DNA torsional elasticity have been considered together. ${ }^{30-32}$ For realistic values of $\sqrt{\left\langle\Delta \Omega^{2}\right\rangle^{27}}$ and of B-DNA elastic twist modulus, $C,^{33-35}$ this theory has shown an attraction between the duplexes with unrelated sequences, although diminished as compared to the homologous ones. ${ }^{25}$ DNA duplexes are soft enough to relax the twist-angle mismatch by twisting the backbones, thereby adjusting the charge patterns and restoring helical "ideality" that brings intermolecular attraction. In dense assemblies, the electrostatic interaction can make DNAs look almost ideally helical, that agrees with what has been experimentally observed. ${ }^{30,36-38}$

Our task is now to incorporate these intermolecular interactions in DNA melting theory.

\section{Model and Basic Equations}

DNA-DNA Interaction in Aggregates. DNA molecules form columnar phases when squeezed by osmotic stress down to interaxial separations of about $30 \AA .{ }^{20,39,40}$ We consider such dense hexagonal aggregates, neglecting for simplicity possible DNA azimuthal frustrations and off-hexagonal arrangements, ${ }^{21,41-44}$ and count only nearest-neighbor DNA-DNA interactions. ${ }^{24,30} \mathrm{We}$ adapt the cylindrical cell model and account for the Donnan equilibrium. ${ }^{30,44,45}$ The value of the renormalized reciprocal screening length in solution between DNAs is then $\kappa \approx \kappa_{\mathrm{D}}\left\{1+(1-\theta)^{2} /\left[\pi h n_{0}\left(R_{\mathrm{s}}^{2}-r^{2}\right)\right]^{2}\right\}^{1 / 4}$. Here, $\kappa_{\mathrm{D}}=$ $\sqrt{8 \pi l_{\mathrm{B}} n_{0}}, l_{\mathrm{B}}$ is the Bjerrum length, $\epsilon \approx 80$ is the dielectric constant of water, ${ }^{46-51} R_{\mathrm{S}}=R \sqrt{\sqrt{3} /(2 \pi)}$ is the cell radius, $r$ is the DNA radius, and $\theta$ is the total fraction of DNA charge neutralization by adsorbed cations. For small $\theta$ values, the Donnan effect results in a substantial accumulation of cations inside DNA aggregates, but at realistic $\theta \approx 0.8$, the difference between $\kappa$ and $\kappa_{\mathrm{D}}$ is not remarkable for not too dense assemblies.

The interaction energy of two duplexes of the length $l$ in parallel juxtaposition has a different form, depending whether they are homologous, nonhomologous rigid or soft ${ }^{21,30,52}$

$$
\begin{gathered}
E_{\text {int }}^{\mathrm{hom}}(l) \approx\left(a_{0}-a_{1}\right) l \\
E_{\mathrm{int}}^{\mathrm{rig}}(l) \approx a_{0} l-2 a_{1} \lambda_{\mathrm{c}}\left[1-\mathrm{e}^{-l /\left(2 \lambda_{\mathrm{c}}\right)}\right] \\
E_{\mathrm{int}}^{\mathrm{soft}}(l) \approx a_{0} l-l a_{1}\left[1-\lambda_{\mathrm{t}} /\left(2 \lambda_{\mathrm{c}}\right)\right]-a_{1} \lambda_{\mathrm{t}}^{2}\left[1-\mathrm{e}^{-l / \lambda_{\mathrm{t}}}\right] /\left(2 \lambda_{\mathrm{c}}\right)
\end{gathered}
$$

the interaction energy of homologous helices grows linearly with the sequence length, whereas the $E_{\text {int }}$ of nonhomologous rigid and soft helices are nonlinear functions of $l$; see Figure 2. We show below that this nonlinearity changes dramatically the picture of DNA melting in assemblies.

The expressions for the interaction harmonics $a_{0,1}(R)$, which decay nearly exponentially with $R$, Figure 1 , are given in Appendix A. The torsional length, $\lambda_{\mathrm{t}}=\sqrt{C /\left(2 a_{1}\right)},{ }^{30}$ character-

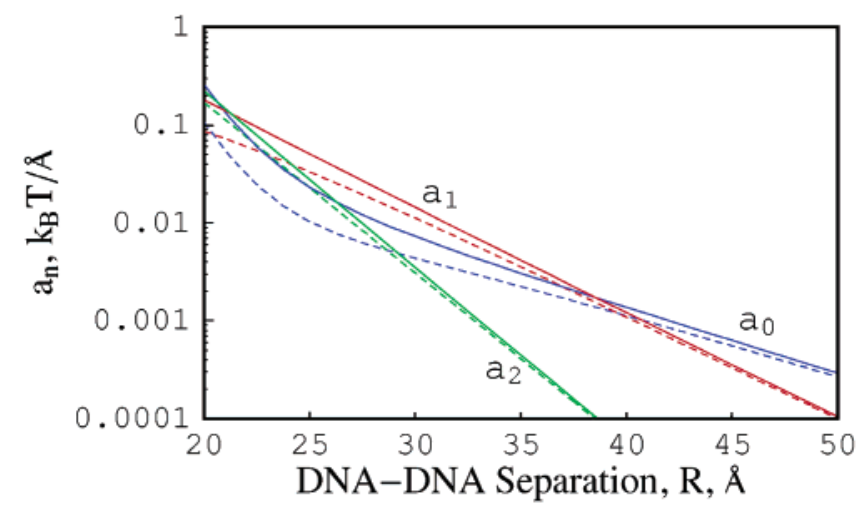

Figure 1. Strength of DNA-DNA interaction. Zeroth $\left(a_{0}\right)$, first $\left(a_{1}\right)$, and second $\left(a_{2}\right)$ helical harmonics of electrostatic interactions between two parallel ideal B-DNAs (solid curves) in solution and in hexagonal aggregate with Donnan effect taken into account (dotted curves), plotted at $f=0.3, \theta=0.8, r=9 \AA$, and $\kappa_{\mathrm{D}}{ }^{-1}=7 \AA$; see Appendix for notations.

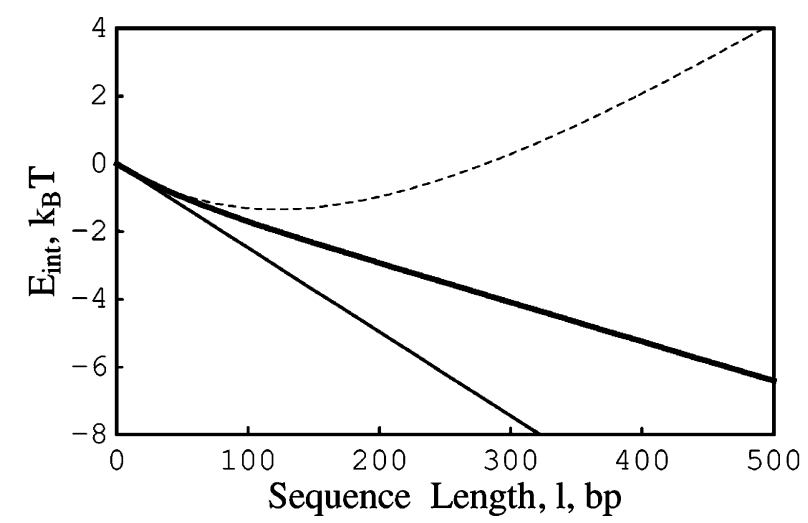

Figure 2. Effect of DNA homology on DNA-DNA electrostatic interactions. The interaction energy of two homologous DNA (thin solid, eq 1a), two nonhomologous rigid helices (dotted, eq 1b), and two nonhomologous soft B-DNA helices (bold solid, eq 1c) at $R=30 \AA$ and $\lambda_{\mathrm{c}}=300 \AA\left(a_{1} \approx 0.0146 k_{\mathrm{B}} T_{\text {room }} / \AA, a_{0} \approx a_{1} / 2\right)$. Other parameters are the same as in Figure 1. The interaction energy of long rigid fragments approaches $a_{0} l-2 a_{1} \lambda_{\mathrm{c}}$.

izes the strength of DNA-DNA interactions relative to the DNA torsional elasticity modulus $C .{ }^{53} \mathrm{We}$ call the helices soft if $\lambda_{\mathrm{t}} /$ $\lambda_{\mathrm{c}} \lesssim 1$ and rigid if $\lambda_{\mathrm{t}} \gg \lambda_{\mathrm{c}}$. The torsional length decreases upon binding of cations in the DNA major groove (this increases $a_{1}$ ), which makes DNA torsional alignments easier and thereby promotes DNA-DNA attraction. The torsional length also decreases with compression of the aggregate, and in very dense assemblies, soft helices appear to be "straightened" by electrostatic interactions and behave almost like ideal duplexes (Figures 3 and $5 \mathrm{c}$ in ref 30 ). For simplicity, we assume a temperatureindependent DNA elastic twist modulus; this assumption likely fails near $T_{\mathrm{m} 0}$, but one can account for $C(T)$ dependence in a more detailed theory.

Melting of a Single DNA. We adopt the simplest, meanfield, two-state, one-dimensional Ising model of DNA melting. ${ }^{4,12}$ The model accounts for the difference in the free energies of bp's in helical $\left(F_{\mathrm{h}}\right)$ and melted $\left(F_{\mathrm{m}}\right)$ states; $F_{\mathrm{m}}-F_{\mathrm{h}}=U-$ $T \Delta S$, where $U$ is the heat of bp melting and $\Delta S$ is the entropy difference between helical and melted states. ( $\Delta S>0$ because melted bp's have more degrees of freedom.) At $T=T_{\mathrm{m} 0}=$ $U / \Delta S$, the numbers of melted $\left(N_{\mathrm{m}}\right)$ and helical $\left(N_{\mathrm{h}}\right)$ bp's are equal, and $s=\exp \left[\left(F_{\mathrm{m}}-F_{\mathrm{h}}\right) /\left(k_{\mathrm{B}} T\right)\right]=1$. With an increase in temperature, the degree of DNA helicity, $N_{\mathrm{h}} /\left(N_{\mathrm{m}}+N_{\mathrm{h}}\right)$, decreases $\left(N_{\mathrm{m}}+N_{\mathrm{h}}=N\right.$, total number of bp). Taking $U=$ $13 k_{\mathrm{B}} T_{\text {room }}$ and $\Delta S=12 k_{\mathrm{B}}{ }^{4}$ gives $T_{\mathrm{m} 0}=50^{\circ} \mathrm{C}$. This model also 
invokes a cooperativity factor $\sigma=\exp \left[-F_{\mathrm{s}} /\left(k_{\mathrm{B}} T\right)\right]$, where $F_{\mathrm{s}}$ is the so-called "stacking energy" required to create a melted region between two intact regions. Smaller $\sigma$ values (larger $F_{\mathrm{s}}$ ) correspond to a more cooperative melting (for B-DNA $\sigma \simeq$ $10^{-4}-10^{-5} 16$ ). No difference in the binding of cations or other condensing agents to helical/melted regions is considered here, but this can be included in the model. ${ }^{4}$ Far below $T_{\mathrm{m} 0}$, the melted fragments are short, and we neglect below various effects of these domains on DNA melting in the aggregate (Discussion).

Melting of DNAs in Hexagonal Aggregates. Various effects may influence DNA melting in aggregates. We assume electrostatic contributions to be dominant in not too dense aggregates, and we try to estimate the shift of $T_{\mathrm{m} 0}$ and the frequency of rupture of double-helical DNA structure separately for aggregates of homologous (those with identical or nearly identical sequences) and nonhomologous (those with completely unrelated sequences) duplexes.

Homologous DNAs. As discussed above, at a high degree of charge neutralization and predominant adsorption of counterions into the major groove, homologous DNA duplexes can attract each other (then $a_{1}>a_{0}$; see Figures 1 and 2). This attraction stabilizes the helical fragments on interacting DNAs and increases the melting temperature. We assume random distribution of helical domains separated by melted sections along the molecules.

The free energy can then be written as the sum of the interaction energy of the helical fragments, the energy of the helix/coil boundaries, the free energy of all DNA base pairs, and the free energy of mixing of helical and melted domains

$$
\begin{array}{r}
F=3 \frac{N_{\mathrm{h}}^{2}}{N} h\left(a_{0}-a_{1}\right)+n F_{\mathrm{s}}+\left(N-N_{\mathrm{h}}\right) F_{\mathrm{m}}+N_{\mathrm{h}} F_{\mathrm{h}}+ \\
k_{\mathrm{B}} T\left\{n \ln \frac{n}{N-N_{\mathrm{h}}}+\left(N-N_{\mathrm{h}}-n\right) \ln \frac{N-N_{\mathrm{h}}-n}{N-N_{\mathrm{h}}}+\right. \\
\left.n \ln \frac{n}{N_{\mathrm{h}}}+\left(N_{\mathrm{h}}-n\right) \ln \frac{N_{\mathrm{h}}-n}{N_{\mathrm{h}}}\right\}
\end{array}
$$

Minimizing $F$ with respect to $n$ (the number of helix/coil boundaries) and $N_{\mathrm{h}}$ at constant $N$, we arrive at two coupled equations

$$
\begin{gathered}
\left(\frac{N-N_{\mathrm{h}}}{n}-1\right)\left(\frac{N_{\mathrm{h}}}{n}-1\right)=\frac{1}{\sigma} \\
\frac{1-n / N_{\mathrm{h}}}{1-n /\left(N-N_{\mathrm{h}}\right)}=s \exp \left\{-\frac{N_{\mathrm{h}}}{N} \frac{6 h\left(a_{0}-a_{1}\right)}{k_{\mathrm{B}} T}\right\}
\end{gathered}
$$

The exponential factor in eq 4 is the only new element added to the theory of melting of single DNA. The attraction between the helical domains within this model can result in a Frumkinlike ${ }^{54} \mathrm{Z}$-shaped "melting isotherm", with the possibility of a first-order phase transition for large enough attraction strength. The critical strength for this transition to occur for a pair of DNAs is approximately $-0.05 k_{\mathrm{B}} T_{\text {room }} / \mathrm{bp}$ (at $F_{\mathrm{s}} \approx 6 \mathrm{kcal} / \mathrm{mol}$ ). This is a realistic value in a sufficiently dense DNA assembly; cf. the value of interaction coefficients in Figure 1. Above this critical strength, i.e., for dense enough aggregates, with the increase of temperature one can expect an abrupt change of the DNA helicity, $N_{2} / N$ (Figure 3 ). This abrupt transition between the stable branches of the melting curve occurs at the temperature marked in Figure 3 by dots, which were obtained using a Maxwell-like equal-area relation in variables $S=-\partial F / \partial T$ and

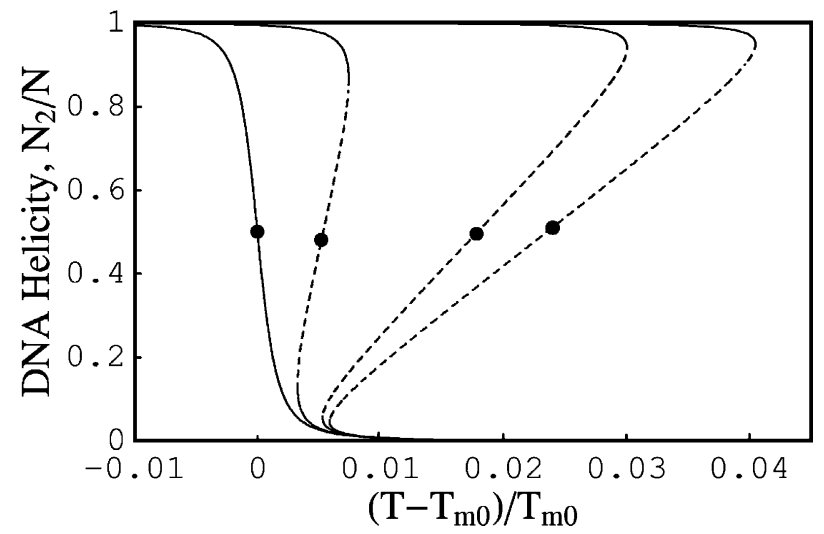

Figure 3. Melting curves for homologous DNAs in hexagonal aggregate, as predicted by eqs 3 and 4, at helix-to-helix separations of $40,30,25$, and $23 \AA$, correspondingly (for the curves from left to right). The unstable branches are the dotted curves. The dots indicate the melting temperature for each curve; for Z-shaped curves an abrupt transition between the stable brunches occurs at this $T$. The parameters are the same as in Figure 1.

$T$. For the parameters chosen in this figure, the transition appears for the aggregates with $R \leq 30 \AA$.

Nonhomologous DNAs. For torsionally rigid duplexes, the position of melted and intact fragments on neighboring DNAs may become correlated in the longitudinal direction to allow DNAs to decrease their interaction energy ("in phase" melting). The degree of correlations is expected to be more pronounced at low $T$, when the mixing entropy of melted and helical fragments is small compared to the interaction energy. The detailed analysis of melting of nonhomologous rigid and soft helices will be presented elsewhere; below we draw the first qualitative predictions.

\section{Results}

Homologous/Ideal Helices. We expect that in the aggregate of homologous DNA duplexes the native state is stabilized by intermolecular attraction; the strength of it per bp can be estimated as $3 h\left(a_{0}-a_{1}\right)<0$. This attraction lowers the bp free energy in the helical state, $F_{\mathrm{h}} \rightarrow F_{\mathrm{h}}+3 h\left(a_{0}-a_{1}\right)$ (eq 4$)$, and thus $T_{\mathrm{m} 0}$ rises by about $3 h\left(a_{0}-a_{1}\right) / \Delta S$. Correspondingly, this correction grows (nearly exponentially) with compression of the aggregate, until such $R$ where $a_{0}(R)-a_{1}(R)$ is minimal. We call this effect electrostatically induced DNA stiffening. Naturally, the shift of $T_{\mathrm{m} 0}$ depends on the parameters used for DNA-DNA interactions and on the aggregate density; it is up to about $8^{\circ} \mathrm{C}$ for the parameters of Figure $1^{55}$ and about 3 times less for a DNA pair "locked in attraction". The average length of DNA helical fragments in the aggregate, $l_{\mathrm{h}}=h N_{\mathrm{h}} / n$, increases as compared to that of free DNA; in the limit of $3 h\left|a_{0}-a_{1}\right| /$ $\left(k_{\mathrm{B}} T\right) \ll 1$, the ratio of these two lengths is $1+3 s h \mid a_{0}-$ $a_{1} \mid\left(k_{\mathrm{B}} T\right)^{-1}\left[(1-s)^{2}+4 s \sigma\right]^{-1 / 2}$, as one can obtain from eqs 3 and 4. Electrostatic DNA-DNA attractive interactions thus prevent melting of homologous helices in assemblies. ${ }^{56}$

Rigid Nonhomologous Helices. Consider the limit of low $T$ with vanishing densities of single-stranded sections, $n / N$, and infinitely large values of $l_{\mathrm{h}} / \lambda_{\mathrm{c}}$. The interaction energy depends nonlinearly on the sequence length, eq $1 \mathrm{~b}$; for long rigid helical fragments, each single-stranded break can lower the energy by about $2 a_{1} \lambda_{c}$, allowing a better azimuthal adjustment of the DNAs. This energy effectively reduces the energy required for the DNA "breaking", $F_{\mathrm{s}}$, making the appearance of melted fragments less costly. The melting transition thus becomes less cooperative. Thus, the density of melted sections on DNA in 
the aggregate compared to that on DNA in solution increases by a factor of $\exp \left[3 \times 2 a_{1} \lambda_{c} /\left(k_{\mathrm{B}} T\right)\right]$; $l_{\mathrm{h}}$ thus diminishes $\exp \left[6 a_{1} \lambda_{c} /\right.$ $\left.\left(k_{\mathrm{B}} T\right)\right]$ times. This effect can be large and grows fast with compression of the aggregate. The DNA-DNA electrostatic interactions thus induce rupture of rigid nonhomologous DNA duplexes. Even far below $T_{\mathrm{m} 0}$, we expect short locally denaturated fragments to appear on interacting rigid DNAs with unrelated sequences. These single-stranded "intermissions" between helical fragments prevent accumulation of twist mismatches and thus allow DNAs to decrease their interaction energy. ${ }^{57}$

This electrostatically induced DNA melting introduces a new mechanism of relaxation of the twist mismatches. The melted fragments here play the same role as the azimuthal kink solitons predicted for long interacting nonhomologous duplexes of a finite rigidity. ${ }^{31}$ We do not consider them here, as when moving closer to $T_{\mathrm{m} 0}$ the impetus for appearance of these solitons disappears.

Soft Nonhomologous Helices. The twist mismatches on juxtaposed DNAs are relaxed via the torsional adjustment/ deformation of the duplexes. Very soft duplexes will always attract each other at optimal azimuthal alignment; for this case, the melting temperature increase is about $3 h \mid a_{0}-a_{1}[1-$ $\left.\lambda_{\mathrm{t}} /\left(2 \lambda_{\mathrm{c}}\right)\right] \mid / \Delta S$, analogous to the homologous helices. (The latter case retrieved by setting $\left.\lambda_{\mathrm{c}}=0.\right)^{58}$

The case of intermediate DNA twist rigidity must be considered in a full analysis, as torsional straightening transitions $^{30,32}$ and melting will compete with each other.

\section{Discussion}

Our main expectations are that (1) the melting temperature of attracting ideal/homologous DNA duplexes should increase with compression of the aggregate and (2) the electrostatic interaction should induce breaking/melting of DNA doublehelical structure on rigid nonhomologous helices.

Limitations of the Model. There are several factors neglected in our model, which may influence the melting behavior. Some of them are related to (i) the chosen simplistic theory of polymer melting; others come from (ii) the neglected modes of interaction between melted sections or from (iii) the peculiarities of DNA duplex-duplex interaction at finite temperatures and the behavior intermediate between being absolutely rigid or absolutely soft. Let us discuss them in some detail.

(i) One may use a more sophisticated theory of melting of a single DNA, accounting for bp heterogeneity and DNA doublestrandedness ${ }^{15}$ as well as for the formation of loops/bubbles in melted regions. ${ }^{15,59,60}$ Note also that the $T_{\mathrm{m} 0}$ of short (several hundreds of bp's) DNAs decreases as compared to that of long helices; ${ }^{13,61}$ short DNAs are easier to unwind from the ends, ${ }^{62}$ and they obey a different theory of melting. ${ }^{13}$

(ii) When the lengths of the melted fragments become substantial (close to and above the melting transition), one can argue that in dense aggregates DNA single strands may undulate. Being confined by neighboring DNAs, this can cause the wellknown entropic repulsion. ${ }^{63-67}$ Also, a release of bound/ condensed counterions upon melting can produce a stronger electrostatic repulsion of DNA phosphates within the melted domains $^{68}$ and disfavor the appearance of melted fragments. These effects can make melted domains unfavorable. This may, in principle, only strengthen conclusion 1 , but it may weaken conclusion 2. However, the difference in melting behavior of homologous and nonhomologous DNAs is likely to be unaffected by these undulations. ${ }^{69}$
Also, due to a confinement of neighboring DNAs, the melted domains in the aggregates have less freedom than in solution. This results in a smaller value of $\Delta S$ and can lead to an increase of $T_{\mathrm{m} 0}$ in assemblies. However, the (entropically favorable) release of the bound water molecules from the melted sections can facilitate the DNA melting (as well as a possible pairing of melted fragments, which is more pronounced in assemblies). ${ }^{9,70}$ All in all, the net effect of the melted pieces on DNA melting in assemblies is not completely clear, and it is not excluded that it may interfere with the predicted effects. However, again, we see no reason this effect should be different for homologous and nonhomologous pairs, and this variance will likely be determined by the effects in the focus of this paper.

(iii) Recently, the electrostatic interaction of DNA duplexes at finite $T$ was considered. ${ }^{32}$ It was shown that the temperatureinduced twist disorder has a similar effect on the interaction energy as the disorder due to DNA nonhomology. This does not affect our qualitative predictions, although results in a weaker attraction between DNA duplexes.

Possible Experiments. Some differences in the melting of 1000-bp-long DNAs in solution and in a liquid-crystalline cholesteric state have been already reported. ${ }^{71}$ It was shown that the melting temperature increases jumpwise by a couple of degrees Celsius with increasing sample density. Thus, intermolecular interactions in the studied assemblies prevent DNA melting. We expect even stronger effects of DNA-DNA interactions on melting in denser, columnar assemblies.

The main new target could be the comparison of the average length of helical fragments and the shift of the melting temperature, for the aggregates of homologous and nonhomologous DNA helices. ${ }^{72}$ Exciting will be to check the effect of the aggregate density, particularly for nonhomologous DNAs. Note that in the region of DNA-DNA separations, where the attraction manifests itself, it is problematic to perform osmotic stress measurements because of an abrupt shrinkage of the DNA assembly. So, a certain range of aggregate densities will not be experimentally accessible.

In experiments, one should ensure that other conditions affecting DNA stability (such as GC content, pattern of adsorbed cations, DNA length, etc.) are kept the same for all of the samples that are going to be compared. More torsionally rigid or softer helices can be considered. Also, the effect of different cations can be monitored in the melting experiments. For example, in the presence of some multivalent cations, which are known to bind into the major DNA groove and induce DNA-DNA attraction, the melting temperature should increase, whereas in solutions of $\mathrm{Ca}^{2+}$ or $\mathrm{Mg}^{2+}$, which have higher affinities to the DNA phosphates and thus do not cause DNA condensation, we do not expect to see this. Particularly interesting will be to study the transition in aggregates of homologous sequences with strong DNA condensing counterions, such as spermine or spermidine, and detect the abrupt melting above certain critical densities of aggregates.

The results, if they agree with theoretical predictions, will give important new information about DNA-DNA interactions and DNA thermal stability in dense aggregates. If not, they will give a hint toward the development of a more involved theory taking into account some of the listed factors ignored in this preliminary consideration.

Acknowledgment. We thank Dominic Lee and Aaron Wynveen for useful discussions. Special thanks are to Sergey Leikin for important criticisms. We thank also the referees for many competent and constructive remarks. The work is a part 
of an EPSRC-funded project (Grant No. GR/S31068/01); A.A.K. acknowledges also the Royal Society Wolfson Merit Research Award.

\section{Appendix A}

The expressions for electrostatic interaction coefficients as functions of DNA-DNA interaxial separation, $R$, $\operatorname{are}^{21,25}$

$$
\begin{gathered}
a_{0}(R)=\frac{8 \pi^{2} \sigma^{2}\left\{\frac{(1-\theta)^{2} K_{0}(\kappa R)}{\epsilon}-\right.}{\kappa^{2}\left[K_{1}(\kappa r)\right]^{2}}- \\
\left.\sum_{p=1}^{2} \sum_{q=-\infty}^{\infty} \frac{[\tilde{f}(p, \theta)]^{2}}{\left.\left.\kappa_{p}^{2}\left[K_{p}^{\prime}\left(\kappa_{p} r\right)\right]^{2}-K_{n} R\right)\right]^{2} I_{q}^{\prime}\left(\kappa_{p} r\right)}\right\} \\
a_{p=1,2}(R)=\frac{16 \pi_{p}{ }^{2} \bar{\sigma}^{2}}{\epsilon} \frac{[\tilde{f}(p, \theta)]^{2}}{\kappa_{p}^{2}} \frac{K_{0}\left(\kappa_{p} R\right)}{\left[K_{p}^{\prime}\left(\kappa_{p} r\right)\right]^{2}}
\end{gathered}
$$

The first term in $a_{0}$ is the interaction of the uniformly charged rods, ${ }^{73}$ whereas the sum of first two $(p=1,2)$ "helical" harmonics describes the image-charge repulsion. The term $a_{1}(R)$ is the first helical harmonic of direct charge-charge interactions.

Here, $\tilde{f}(p, \theta)=f \theta+(-1)^{p} \theta(1-f)-\cos \left(p \tilde{\phi}_{\mathrm{s}}\right) ; I_{p}, K_{p}, I_{p}^{\prime}$, and $K_{p}^{\prime}$ are, respectively, the modified Bessel functions and their derivatives; $r=(9 \AA)$ is the DNA radius; $\kappa_{\mathrm{D}}^{-1}$ is the Debye screening length in the bulk solution; $\epsilon=80$ is the solvent dielectric constant; $\kappa_{p}=\sqrt{\kappa^{2}+p^{2}(2 \pi / H)^{2}}$ is the effective reciprocal screening length of the $p$ th DNA-DNA interaction harmonics; $H$ is the average DNA pitch; $\bar{\sigma}=16.8 \mu \mathrm{C} / \mathrm{cm}^{2}$ is the mean surface charge density of DNA phosphates; $\tilde{\phi}_{\mathrm{s}}(\sim 0.4 \pi$ for B-DNA) is the azimuthal half-width of the B-DNA minor groove; $\theta$ is the fraction of DNA charge compensated for by adsorbed cations; $f$ and $(1-f)$ are the fractions of adsorbed cations in minor and major DNA groove, respectively. (There are no adsorbed cations on the phosphate strands.)

The phosphate strands and the cations adsorbed in the grooves are considered here as infinitely thin charged spirals. One can also account for a more realistic, thermally smeared, charge distribution that would slightly weaken the electrostatic interactions of helices. ${ }^{30} \mathrm{We}$ assume that the adsorption of cations on DNA is irreversible; no changes in adsorption occur with temperature ( $f$ and $\theta$ are temperature-independent; for adjustable patterns of adsorbed cations on DNAs, see ref 24). We consider not too dense aggregates, $R>25 \AA$, where the effects of DNA azimuthal frustrations ${ }^{30,43,44}$ may be neglected. (We set $a_{2}=$ 0 .) We use the value of the macroscopic dielectric constant in solution between DNAs, $\epsilon=80$; see ref 46 .

\section{References and Notes}

(1) Franklin, R. E.; Gosling, R. G. Nature 1953, 171, 740. Watson, J. D.; Crick, F. H. C. Nature 1953, 171, 737. Wilkins, M. H. F.; Stokes, A R.; Wilson, H. R. Nature 1953, 171, 738.

(2) Saenger, W. Principles of Nucleic Acid Structure; SpringerVerlag: New York, 1984.

(3) Eichhorn, G. L.; Shin, Y. A. J. Am. Chem. Soc. 1968, 90, 7323

(4) Vedenov, A. A.; Dykhne, A. M.; Frank-Kamenetskii, M. D. Sov. Phys. Usp. 1972, 14, 715

(5) Berestetskaya, I. V.; Frank-Kamenetskii, M. D.; Lasurkin, Yu. S. Biopolymers 1974, 13, 193.

(6) Wang, W. D.; Yin, H.; Landick, R.; Gelles, J.; Block, S. M. Biophys. J. 1997, 72, 1335. Strick, T. R.; Croquette, V.; Bensimon, D. Proc. Natl. Acad. Sci. U.S.A. 1998, 95, 10579. Rouzina, I.; Bloomfield, V. A. Biophys. J. 2001, 80, 882 .
(7) Hwa, T.; Marinari, E.; Sneppen, K.; Tang, L. Proc. Natl. Acad. Sci. U.S.A. 2003, 100, 4411.

(8) Danilowicz, C.; Coljee, V. W.; Bouzigues, C.; Lubensky, D. K.; Nelson, D. R.; Prentiss, M. Proc. Natl. Acad. Sci. U.S.A. 2003, 100, 1694

(9) Duguid, J. G.; Bloomfield, V. A.; Benevides, J. M.; Thomas, G. J., Jr. Biophys. J. 1995, 69, 2623.

(10) Duguid, J. G.; Bloomfield, V. A.; Benevides, J. M.; Thomas, G. J., Jr. Biophys. J. 1995, 69, 2642.

(11) Different interpretations of this effect have been discussed in the literature. In particular, these ions have been speculated to cross-link the melted bp's on neighboring DNAs, promoting DNA aggregation. Shibata J. H.; Schurr J. M. Biopolymers 1981, 20, 525.

(12) Peller, L. J. Phys. Chem. 1959, 63, 1194.

(13) Poland, D.; Sheraga H. A. Theory of Helix-Coil Transition in Biopolymers; Academic Press: New York, London, 1970.

(14) Zimm, B. H.; Rice, S. A. Mol. Phys. 1960, 3, 391. Lifson, S.; Zimm, B. H. Biopolymers 1963, 1, 15. Crothers, D. M. Biopolymers 1968, 6, 1391.

(15) Lasurkin, Yu. S.; Frank-Kamenetskii, M. D.; Trifonov, E. N. Biopolymers 1970, 9, 1253 and references therein.

(16) Crothers, D. M.; Zimm, B. H. J. Mol. Biol. 1964, 9, 1.

(17) Also, altering the $\mathrm{pH}$ is known to increase the net charge on polypeptides that could cause additional disruption of their intramolecular hydrogen bonds. ${ }^{12}$ Another example is the folding of a polypeptide chain on itself upon melting, facilitated by hydrophobic interactions of helical units; as a result, the melting temperature rises.

(18) Pincus, P.; de Gennes, P. G. J. Polym. Sci., Polym. Symp. 1978 $65,85$.

(19) Rau, D. C.; Lee, B.; Parsegian, V. A. Proc. Natl. Acad. Sci. U.S.A 1984, 81, 2621. Strey, H. H.; Parsegian, V. A.; Podgornik, R. Phys. Rev. Lett. 1997, 78, 895 .

(20) Rau, D. C.; Parsegian, V. A. Biophys. J. 1992, 61, 246. Rau, D. C.; Parsegian, V. A. Biophys. J. 1992, 61, 260.

(21) Kornyshev, A. A.; Leikin, S. J. Chem. Phys. 1997, 107, 3656 Kornyshev, A. A.; Leikin, S. 1998, 108, 7035 (erratum).

(22) Kornyshev, A. A.; Leikin, S. Proc. Natl. Acad. Sci. U.S.A. 1998 95,13579

(23) Kornyshev, A. A.; Leikin, S. Biophys. J. 1998, 75, 2513.

(24) Cherstvy, A. G.; Kornyshev, A. A.; Leikin, S. J. Phys. Chem. B 2002, 106, 13362

(25) Kornyshev, A. A.; Leikin, S. Phys. Rev. Lett. 1999, 82, 4138.

(26) Kornyshev, A. A.; Leikin, S. Phys. Rev. Lett. 2001, 86, 3666.

(27) Kabsch, W.; Sander, C.; Trifonov, E. N. Nucleic Acids Res. 1982 10,1097 .

(28) Gorin, A. A.; Zhurkin, V. B.; Olson, W. K. J. Mol. Biol. 1995 247,34

(29) Olson, W. K.; Gorin, A. A.; Lu, X.-J.; Hock, L. M.; Zhurkin, V. B. Proc. Natl. Acad. Sci. U.S.A. 1998, 95, 11163.

(30) Cherstvy, A. G.; Kornyshev, A. A.; Leikin, S. J. Phys. Chem. B 2004, 108, 6508 .

(31) Kornyshev, A. A.; Wynveen, A. Phys. Rev. E 2004, 69, 041905.

(32) Lee, D. J.; Wynveen, A.; Kornyshev, A. A. Phys. Rev. E 2004, 70, 051913.

(33) Crothers, D. M.; Drak, J.; Kahn, J. D.; Levene, S. D. DNA Bending, Flexibility and Helical Repeat by Cyclization Kinetics. In Methods in Enzymology; Lilley, D. M. J., Dahlberg, J. E., Eds.; Academic Press: San Diego, 1992; Vol. 212B, p 3.

(34) Shore, D.; Baldwin, R. J. Mol. Biol. 1983, 170, 957. FrankKamenetskii, M. D.; Lukashin, A. V.; Anshelevich, V. V.; Vologodskii,

V. V. J. Biomol. Struct. Dyn. 1985, 2, 1005.

(35) Vologodskii, A. V.; Anshelevich, V. V.; Lukashin, A. V.; FrankKamenetskii, M. D. Nature 1979, 280, 294.

(36) Langridge, R.; Wilson, H. R.; Hooper, C. W.; Wilkins, M. H. F.; Hamilton, L. D. J. Mol. Biol. 1960, 2, 19

(37) Rhodes, D.; Klug, A. Nature 1980, 286, 573.

(38) Zimmerman, S. B.; Pheiffer, B. H. Proc. Natl. Acad. Sci. U.S.A 1979, 76, 2703

(39) Rau, D. C.; Lee, B. K.; Parsegian, V. A. Proc. Natl. Acad. Sci. U.S.A. 1984, 81, 2621.

(40) Rau, D. C.; Parsegian, V. A. Science 1990, 249, 1278. Leikin, S. Rau, D. C.; Parsegian, V. A. Nat. Struct. Biol. 1995, 2, 205. Bonnet-Gonnet, C.; Leikin, S.; Chi, S.; Rau, D. C.; Parsegian, V. A. J. Phys. Chem. B 2001 105,1877

(41) Strey, H. H.; Wang, J.; Podgornik, R.; Rupprecht, A.; Yu, L.; Parsegian, V. A.; Sirota, E. B. Phys. Rev. Lett. 2000, 84, 3105.

(42) Lorman, V.; Podgornik, R.; Zecks, B. Phys. Rev. Lett. 2001, 87 , 218101

(43) Wynveen, A.; Lee, D. J.; Kornyshev, A. A. Eur. Phys. J. E. 2005, 16,303

(44) Harreis, H. M.; Kornyshev, A. A.; Likos, C. N.; Löwen, H.; Sutmann, G. Phys. Rev. Lett. 2002, 89, 018303.

(45) Cherstvy, A. G. J. Phys: Condens. Matter 2005, 17, 1363

(46) We do not consider here the effects of nonlocal water polarizability ${ }^{47}$ and nonlocal electrostatics ${ }^{48}$ that may lead to a reduction of $\epsilon$ in a confined 
space. Nonlinear effects (dielectric saturation) 49,50 $^{4}$ and $\epsilon(T)$ dependence ${ }^{51}$ are also neglected as well as the effect of the Donnan equilibrium on dielectric screening. Thus, we treat not too dense assemblies and neglect the dependence of $\epsilon$ on the aggregate density.

(47) Kornyshev, A. A. Nonlocal Electrostatics of Solvation. In The Chemical Physics of Solvation; Dogonadze, R. R., Kalman, E., Kornyshev, A. A., Ulstrup, J., Eds.; Elsevier: Amsterdam, 1985; Part A, Chapter 3, p 77.

(48) Vorotyntsev, M. A.; Kornyshev, A. A. Electrostatics of Media with the Spatial Dispersion; Nauka: Moscow, 1993.

(49) Liszi, J.; Ruff, I. Semi-Macroscopic Models of Ionic Solvation. In The Chemical Physics of Solvation; Dogonadze, R. R., Kalman, E., Kornyshev, A. A., Ulstrup, J., Eds.; Elsevier: Amsterdam, 1985; Part A, Chapter 4, p 119.

(50) Kornyshev, A. A.; Sutmann, G. Phys. Rev. Lett. 1997, 79, 3435. Kornyshev, A. A.; Sutmann, G. J. Electroanal. Chem. 1998, 450, 143.

(51) CRC Handbook of Chemistry and Physics, 75th ed.; Lide, D. R., Ed.; CRC Press: Boca Raton, FL, 1994.

(52) Cherstvy, A. G. Interaction, Recognition, and Condensation of DNA Duplexes. Ph.D. Thesis. Düsseldorf University, 2002.

(53) The torsional length $\lambda_{\mathrm{t}}$ should not be confused with the DNA torsional persistence length, $\lambda_{\mathrm{p}}=2 C /\left(k_{\mathrm{B}} T\right)$ (Another, more common, notation for the DNA twist rigidity constant is $\left.\tilde{\lambda}_{\mathrm{p}}=C /\left(k_{\mathrm{B}} T\right) \approx 750 \AA\right)$. Schurr, M. J. Biopolymers 1985, 24, 1233.

(54) Adamson, A. W.; Gast, A. P. Physical Chemistry of Surfaces; Wiley-Interscience Publication: New York, 1997.

(55) As the adsorption of cations in the major groove and a better neutralization of the DNA phosphates strengthen the DNA-DNA electrostatic attraction, ${ }^{25}$ our model predicts a larger rise of $T_{\mathrm{m} 0}$ for such DNAs (For instance, at $\theta=0.9$ and $f=0$, the rise of the melting temperature can be up to $\sim 30-40{ }^{\circ} \mathrm{C}$.) Note here that about $90 \%$ of the phosphate charges are typically neutralized upon DNA condensation with multivalent counterions, which often adsorb in the DNA major groove. Bloomfield, V. A. Biopolymers 1997, 44, 269.

(56) Even for conditions favoring DNA-DNA electrostatic attraction, the region of image-force repulsion emerges at distances of several angstroms between the DNA surfaces ${ }^{21}$ (Figure 1). The repulsion of net negatively charged DNAs prevails also at large DNA-DNA interaxial separations; see Figure 7 in ref 24 . To get a plausible estimate for the shift of $T_{\mathrm{m} 0}$ for the case of DNA-DNA repulsion in the aggregates, one needs also to treat interactions of melted fragments neglected in the present study, including the effects outlined in the second paragraph of section $\mathrm{V}$.

(57) Cherstvy, A. G.; Kornyshev, A. A.; Leikin, S., to be submitted for publication.

(58) For soft nonhomologous helices, one can also expect a slight increase in the concentration of single-stranded domains, which can lower the (nonlinear) interaction energy of such fragments; see eq 1c. Note also that since the interaction energy of very short rigid, soft, and homologous DNA fragments is the same, Figure 2, above the melting transition, when the helical domains become short, the electrostatic interactions is expected to modify the melting curves in a similar manner for all three cases.

(59) Peyrard, M.; Bishop, A. R. Phys. Rev. Lett. 1989, 62, 2755. R44.

(60) Dauxois, T.; Peyrard, M.; Bishop, A. R. Phys. Rev. E 1993, 47,

(61) Crothers, D. M.; Kallenbach, N. R.; Zimm, B. H. J. Mol. Biol. $1965,11,802$.

(62) Zeng, Y.; Montrichok, A.; Zocchi, G. J. Mol. Biol. 2004, 339, 67.

Zeng, Y.; Montrichok, A.; Zocchi, G. Phys. Rev. Lett. 2003, 91, 148101.

(63) Israelachvili, J. N. Intermolecular and Surface Forces; Academic Press: London, 1985

(64) Odijk, T. Biophys. Chem. 1993, 46, 69. Odijk, T. Europhys. Lett. 1993, 24, 177.

(65) Odijk, T. Macromolecules 1983, 16, 1340.

(66) Kassapidou, K.; van der Maarel, J. R. C. Eur. Phys. J. B 1998, 3, 471.

(67) Burkhardt, T. W. J. Phys. A 1995, 28, L629.

(68) To account for this repulsion, one can use the energy (eq A1) without the second term in curly brackets that corresponds to the interaction of the image charges, since the single-stranded DNAs loose the lowdielectric core considered for the interaction of the double-stranded DNA

(69) First, the helical fragments on one DNA are azimuthally and translationally confined by interactions with the fragments on neighboring DNAs in dense aggregates. (In moderately dense DNA fibers, of course, the azimuthal/twist DNA motions are not necessarily frozen.) Second, since the melted fragments relax the mismatch accumulated in the helical fragments, a braiding of the melted fragments is expected to occur. These two factors are likely to diminish the free energy of entropic repulsion between long single-stranded domains. Without these constraints, the latter would scale as $l_{\mathrm{p}}{ }^{-1 / 3} R^{-2 / 3},{ }^{64}$ where $l_{\mathrm{p}}$ is the persistence length of singlestranded DNA $(\sim 20 \AA)$. If this entropic repulsion occurs in reality, then such scaling can be extracted from experimental data. Below the melting transition, however, when the melted domains are very short, this entropic repulsion in the aggregates can be neglected.

(70) Yakubovskaya, M. G.; et al. Eur. J. Biochem. 2001, 268, 7.

(71) Grasso, D.; Fasone, S.; La Rosa, C.; Salyanov, V. Liq. Cryst. 1991, 9, 299.

(72) For DNA in solution, the length of the helical fragments and the concentration of single-stranded breaks/defects in DNA structure can be measured experimentally and treated theoretically, see ref 15 . [The measurement of the intrinsic viscosity of partially melted DNA together with the degree of DNA helicity provides the mean length of the helical domains (flexible single-stranded fragments decrease the DNA persistence length). The number of melted fragments has been obtained by measuring the initial velocity of stimulated DNA unwinding under the action of formaldehyde.] In dense DNA assembly, however, different methods have to be used.

(73) Brenner, S. L.; Parsegian, V. A. Biophys. J. 1974, 14, 327. 